\title{
Risk analysis of power systems for both real and reactive power
}

\author{
Wenping QIN, Peng WANG (ه), Xiaoqing HAN, \\ Fanhua MENG
}

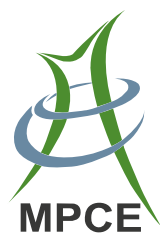

\begin{abstract}
With the high penetration of renewable energy sources, the reliability of power systems becomes more vulnerable than ever because of the greater uncertainty and intermittence in power generation. Reactive power plays an important role in the power system reliability, because it is closely related to the system voltage stability and voltage collapse. However, reactive power-related reliability issues are seldom emphasized in conventional power reliability evaluations. This article investigates power system reliability of real and reactive power. Real and reactive power shortages and the associated voltage violations due to system failures are considered on reliability evaluation of power systems. A three-stage load-shedding technique for post contingencies is implemented to determine the contributions of real and reactive power on the system reliability and to find an optimal way to release network violation. The results provide the detailed information on power system planning and operation for system planners and operators from real and reactive power aspects.
\end{abstract}

Keywords Reactive power, Risk analysis, Reliability, Voltage stability

\section{Introduction}

Voltage collapse usually occurs in heavy load power systems. A heavy load power system subject to a failure or

Received: 18 October 2012/Accepted: 8 April 2013/Published online: 7 September 2013

(C) The Author(s) 2013. This article is published with open access at Springerlink.com

W. QIN, P. WANG, X. HAN, F. MENG, Taiyuan University

of Technology, Taiyuan 030024, China

W. QIN

e-mail: qinwenping1027@163.com

P. WANG, School of EEE, Nanyang Technological University, Singapore 639798, Singapore

$(\bowtie)$ e-mail: epwang@ntu.edu.sg disturbance undergoes voltage collapse if post-disturbance equilibrium voltages are below acceptable limits. The voltage collapse is generally initiated by either load variations or contingencies and is characterized by high reactive power demand and high reactive power loss in transmission network and shortage of fast-acting reactive reserves [1]. The fast reactive sources are generators, synchronous condensers, and power electronics-based flexible dynamic voltage restorer. Adequate reactive power reserve is expected to maintain system integrity during post contingency operation when considering random failures. As a well-established ancillary service, the reactive power support and the voltage control play a vital role in power system operation. The impact of reactive power on system stability and security has been well investigated [2-6]. During a contingency, the real power component of line loading does not change significantly, whereas the reactive power flow can change dramatically [2]. The reason is that the bus voltage drop due to a component failure reduces the reactive power generation from the charging of line and shunt capacitors. Therefore, sufficient reactive reserve should be available to meet the reactive power requirement following a contingency. Reactive power which can be delivered in a power system depends on its network configuration, operating condition, and locations of reactive power sources. References [2-6] show that the reactive power was the key to solve voltage problems in system operation and should be considered in reliability evaluation.

Recently, renewable energy sources (RESs), such as solar and wind energy have been widely integrated into power systems. Due to the fluctuation and unpredictable characteristics of RESs and the system load, the system voltage stability issues become more complex. The high penetration of wind energy has the potential to change the modes of instability in power systems [7]. Reference [7] shows that an increase in wind penetration resulted in 
greater demand on reactive power, which may lead to voltage instability if not met by the existing power system. In distribution systems, the photovoltaic (PV) power fluctuation combined with the system tap changer control scheme and mechanism is closely related to an unaccepted voltage drop [8]. Therefore, it is important to investigate the impact of reactive power on reliability of power systems with RESs.

However, conventional reliability evaluations of power systems concern more on real power adequacy problems [9-12]. The reactive power-related reliability issues have not been separately studied in detail. The proposed reliability indices in most reliability evaluation techniques are related to adequacy aspect, such as the expected energy not supplied (EENS), expected load curtailment (ELC), etc. First, those indices are usually calculated using the proportional active load-shedding techniques. Second, system unreliability caused by reactive power shortage is hidden by those indices. Under-voltage load shedding is the least and lowest cost solution to severe voltage problems [13]. Some under-voltage load-shedding strategies including real and reactive power load curtailments are adopted to mitigate system voltage instability [13-18] in real-time operation of power systems. It is a challenge to implement those techniques on reliability evaluation.

Reference [19] proposed a technique to evaluate reliability indices which take into account both real and reactive power shortages due to failures caused by real and reactive power sources, such as generators, synchronous condensers, compensators, and flexible alternative current transmission system (FACTS). Reactive power shortage and its associated voltage violations due to failures of reactive power sources were considered. Some new reliability indices represented the impact of reactive power shortage on system reliability. The reliability indices due to reactive power shortages were separated from those due to real power shortages.

This article investigates both real and reactive power shortages and the associated voltage violations due to system failures on reliability evaluation. The load point indices related to real and reactive power shortages are defined. The real power shortage and the network violations of a contingency are released using a three-stage loadshedding process instead of the two-stage process in [19]. The proposed load-shedding technique during post contingencies is implemented to illustrate the importance of the reactive power on system reliability and to find the most economical way to release network violation. The contributions of real and reactive power to the load point and system reliability are clearly decoupled using the proposed technique.

Section 2 reviews the voltage stability issues related to the reliability. Section 3 presents load point and system reliability indices related to real and reactive power. In Section 4, different load-shedding strategies and reactive power issues related to power system reliability are discussed. The equivalent $220 \mathrm{kV}$ Taiyuan system of Shanxi Province in China is analyzed to illustrate the technique and the results are presented in Section 5. Section 6 concludes the article.

\section{Voltage stability and reliability}

A power system from a load bus can be represented by an equivalent generator on power system analysis. Real power $P$ and reactive power $Q$ transferred from a lossless power system to a load bus had been described clearly in [1]. The load bus voltage $V$ is the function of $P$ and $Q$.

The relationship between the real power and the voltage is usually represented by a normalized $p v$ curve. The $p v$ curve for a constant power factor can be obtained by projecting the intersection curve of $V(P, Q)$ and the vertical plane $Q=P \operatorname{tg} \varphi$. The $p v$ curves for the four different $\operatorname{tg} \varphi$ are shown in Fig. 1. Curves 1, 2, and 3 correspond to inductive loads with lagging power factors. Curve 4 corresponds to capacitive load with leading power factor. It can be seen from the figure that the more real power load can be supplied without violating the voltage limitation $v_{\min }$ as power factor increases. Voltage reduces as real power load increases for a given $\operatorname{tg} \varphi$. When $p$ reaches to its maximum value $p_{\max }$, the voltage collapses. Taking Curve 3 as an example, the bus voltage is normal at point a, the voltage at point $b$ reaches to the lowest limit, whereas the reliability margin to voltage collapse at point $\mathrm{c}$ is the minimum. A leading power factor will cause overvoltage problem, as shown in Curve 4.

The relationship between the reactive power and the voltage is usually represented by a normalized $v q$ curve. The $v q$ curves for three different values of $p$ are shown in Fig. 2. For a given $p=0.25$, there are two mathematical

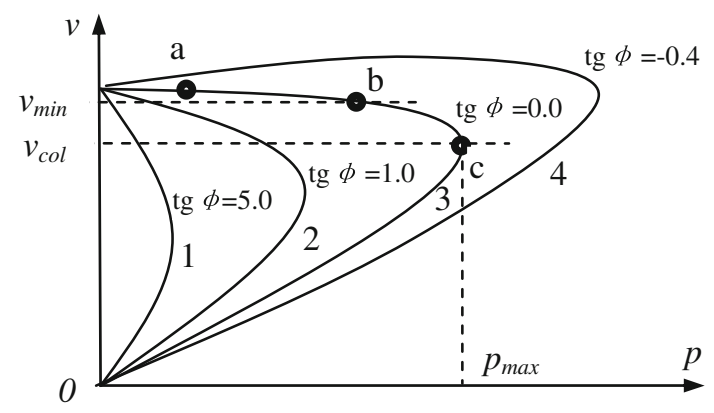

Fig. $1 p v$ curves for different power factors with a given $Q$ 


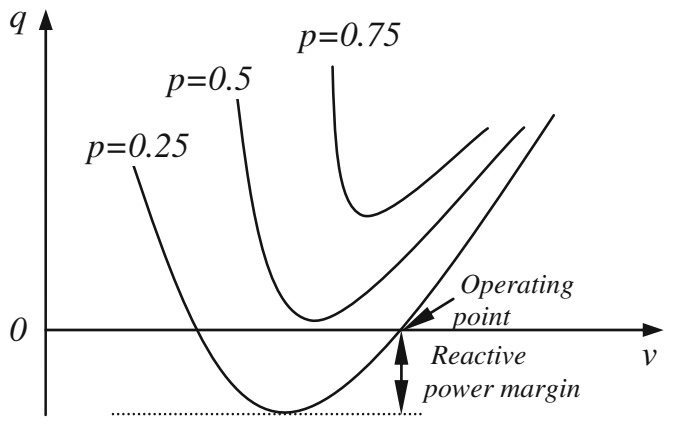

Fig. $2 v q$ curves for different $p$ with a constant power factor

voltage solutions. Only voltage at right-hand side is realistic. The realistic voltage increases with $q$. The reactive power distance from the operating point to the bottom of the curve is the reactive power margin.

Voltage stability has the system ability to maintain voltage so that both power and voltage are controllable when the load admittance increases or load power increases [20]. The lack of adequate real and reactive power resources during system heavy load conditions and severe system contingencies has been recognized as a major factor in a voltage collapse process. Voltage collapse is a dynamic and normally large disturbance phenomenon [1]. System voltage collapses when one bus reaches its collapse point [21]. When a failure occurs in a power system, the impedance $X$ of the equivalent generator from a bus will change, and the maximum real and reactive power supplied by the system will also be affected. Therefore, system voltage collapse point will also change. The large area blackouts are initialized by the voltage collapse at one or more buses during a system failure. Therefore, it is a basic reliability requirement to prevent the system from the voltage collapse point under various operating conditions and contingencies through providing sufficient contingency real and reactive power reserves and curtailing the real and reactive power load in the worst scenarios.

\section{Reliability indices and evaluation technique}

Conventional reliability indices provided by most reliability evaluation techniques are only related to the real power, such as EENS and ELC. Those indices hide some important system reliability problems which are closely related to voltage stability. In order to provide comprehensive information on system reliability from different aspects for system planners and operators, the reliability indices related to real and reactive power shortages [19] are used in this article. Those indices represent different aspects of power system reliability in detail.
The EENS for bus $j$ due to the real power shortage $E E N S_{P j}$ and the reactive power shortage $E E N S_{Q j}$ are defined as follows:

$\operatorname{EENS}_{P j}=\sum_{i=1}^{N C} L C_{P i j} \times p_{i} \times 8,760$,

$E E N S_{Q j}=\sum_{i=1}^{N C} L C_{Q i j} \times p_{i} \times 8,760$,

where $N C$ is the total number of considered contingencies, $L C_{P i j}$ and $L C_{Q i j}$ are the real load curtailments at bus $j$ due to real and reactive power shortages for state $I$, respectively.

The expected reactive power not supplied for bus $j$ due to the real power shortage $E V N S_{P j}$ and the reactive power shortage $E V N S_{Q_{j}}$ are defined as

$E V N S_{P j}=\sum_{i=1}^{N C} Q C_{P i j} \times p_{i} \times 8,760$,
$E V N S_{Q j}=\sum_{i=1}^{N C} Q C_{Q i j} \times p_{i} \times 8,760$,

where $Q C_{P i j}$ and $Q C_{Q i j}$ are the reactive load curtailments at bus $j$ due to real and reactive power shortages for state $i$, respectively.

The expected reactive power shortage for bus $j$ due to the voltage violation $E \operatorname{Var} S_{j}$ is defined as

$E \operatorname{Var} S_{j}=\sum_{i=1}^{N C} \operatorname{VarS}_{Q i j} \times p_{i} \times 8,760$,

where $\operatorname{Var} S_{Q i j}$ is the active power shortage which causes voltage violation for state $i$, it is the reactive power which should be injected at bus $j$ to relieve the bus voltage violation.

The system indices can be calculated from the load point indices. For example, the system $E E N S_{P}$ and $E E N S_{Q}$ can be calculated as

$\operatorname{EENS}_{P}=\sum_{j=1}^{N L} \operatorname{EENS}_{P j}$,

$E E N S_{Q}=\sum_{j=1}^{N L} E E N S_{Q j}$

where $N L$ is the number of buses in the system. Other system indices can be calculated using similar formulas.

The contingency enumeration technique is used to determine the reliability indices. The power flow technique will be used to determine network violations for each contingency. Different load-shedding techniques and reactive power injection approaches will be used to release network violations and the related $L C_{P i j}, Q C_{P i j}, L C_{Q i j}, Q C_{Q i j}$ and $\operatorname{Var} S_{Q i j}$. 


\section{Load-shedding technique}

\subsection{Real power load shedding}

Real power load shedding has been used in most conventional reliability techniques to maintain real power balance caused by the real power shortage for a system contingency. The proportional load-shedding scheme is usually used to cut the load at each bus based on its percentage of the total system load. The priority load shedding and reliability cost/worth-based load-shedding [22] techniques are used to curtail the real power load based on customer's willingness to pay for their reliability. Those load-shedding techniques and the resulting indices are usually used in generation planning.

\subsection{Reactive power characteristics in network}

There are three aspects of differentiating reactive power from active power in power system operation, and they should be considered in reliability evaluation. First, it is not efficient to transfer reactive power over a long distance because reactive power losses in transmission lines are significant and the bus voltage is very sensitive to reactive power. Therefore, the reactive power shortage is usually compensated locally in weakly connected grids. Second, the major role of reactive power is to maintain voltage stability/ security of power systems. Therefore, the impact of reactive power on system reliability in terms of energy not supplied is indirect and is calculated based on the reactive power shortage and voltage violations. Finally, the reactive power losses change with system configuration and operation conditions [23, 24]. Reactive power requirements for voltage restoration after a contingency are heavily dependent on reactive power reserve distributions in a power system. In order to reasonably determine the real and reactive power dispatch and post contingency load shedding, the characteristics of real and reactive power corresponding to bus voltage and their correlation have to be considered.

\subsection{Real and reactive power load shedding}

A three-stage load-shedding process is adopted to distinguish the reliability indices due to the reactive power shortage from those caused by the real power shortage. The objective is to provide detailed information on current weakness of real and reactive power resources and future system expansion for system planners and operators.

In the first stage, the real power capacity including both generation and reserve of the total available system is compared with the real power demand including the total real power load and transmission losses of the total system.
If the total available capacity is less than the total load plus loss, then real power loads at all the load buses are curtailed in the system range using different load-shedding techniques [22]. Reactive power load at each bus is also curtailed correspondingly based on the initial power factor. The proportional load shedding is used in this stage to illustrate the technique. $L C_{P i j}$ and $Q C_{P i j}$ at bus $j$ for contingency state $i$ are calculated as follows:

$L C_{P i j}=P_{l j}\left|\sum_{g=1}^{N G_{i}} P_{g}-\sum_{j=1}^{N L} P_{l j}-P_{i}^{\text {loss }}\right| / \sum_{j=1}^{N L} P_{l j}$,

$Q C_{P i j}=L C_{P i j} \operatorname{tg} \phi_{j}$,

where $N G_{i}$ is the number of generator units for state $I, P_{g}$ is the maximum available real power capacity of unit $g, P_{l j}$ is the real power load at bus $j$ for the normal state, $P_{i}^{\text {loss }}$ is the estimated system real loss for the normal state, and $\phi_{j}$ is the power factor angle at bus $j$.

In the second stage, the $Q$ shortage problem of system range is investigated based on $Q$ generation at the swing bus. The system $Q$ shortage is determined based on the load and generation conditions after the first-stage load-shedding using AC power flow analysis. $Q$ compensations at $P V$ buses are first checked. A $P V$ bus at which the $Q$ compensation is over or equal to its limit will be changed into $P Q$ bus to fix its reactive power compensation to the limit. AC power flow is again performed based on the fixed $Q$ compensations at $P Q$ buses. If the $Q$ generation at the swing bus is in excess of its limit, then the proportional load shedding is used in this stage until system $Q$ is balanced. $L C_{Q i j}$ and $Q C_{Q i j}$ at bus $j$ for contingency state $i$ are calculated as follows:

$Q C_{Q i j}=Q_{l j}\left(Q_{\mathrm{s}}-Q_{\mathrm{s}}^{\max }\right) / \sum_{j=1}^{N L} Q_{l j}$,

$L C_{Q i j}=Q C_{Q i j} / \operatorname{tg} \phi_{j}$,

where $Q_{s}$ and $Q_{s}^{\max }$ are the actual $Q$ generation and its limit at the swing bus, respectively, $Q_{l j}$ is the reactive power load at bus $j$ for the normal state.

In the third stage, the voltage problem at buses is checked using $\mathrm{AC}$ power flow analysis. The bus voltage less than the voltage set point means the local reactive power shortage. Because of the low efficiency of delivering reactive power over a long distance, load shedding is performed at nodes with the voltage violations. Both real and reactive power loads are iteratively curtailed in steps of $1 \%$ with the fixed power factor until the voltage violation is eliminated. The detailed load-shedding process in this stage for contingency state $i$ is shown in Fig. 3. It is noted that if the voltage violation at those buses still exists after loads completely curtailed, then it is necessary to cut the loads at their adjacent nodes according to local characteristics of the reactive power. 


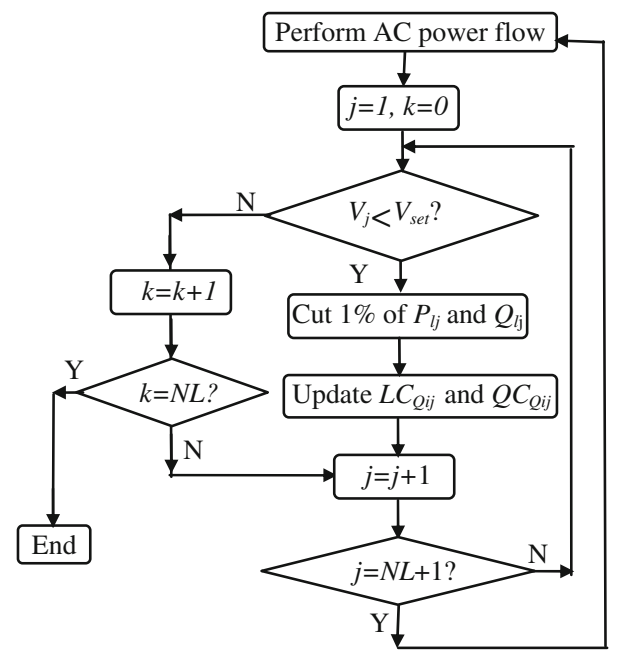

Fig. 3 Flow chart of the third-stage load-shedding process for state $i$

\subsection{Reactive power injection}

The voltage violations related to reactive power shortage $\operatorname{Var} S_{Q i j}$ can be solved using local reactive injection method. In this method, reactive power is injected at the nodes with the voltage violations to restore the voltage. When the voltage reaches the voltage set point, the corresponding reactive power injected is the reactive shortage $\operatorname{Var} S_{Q i j}$. It should be noted that the impact of reactive power injection on bus voltage is very sensitive to network configuration and reactive power source distribution. It is also related to the economic considerations of the consumers. In this article, the reactive power is gradually injected in step of $1 \%$ of the reactive load at a bus with the voltage violation until the voltage problem is solved. The objective of reactive power injection is to provide additional information for system operators and planners to add new reactive power sources in future planning and operation.

\section{System study}

The simplified $220 \mathrm{kV}$ Taiyuan power system in Shanxi Province of China as shown in Fig. 4 is used to illustrate the proposed technique.

The system has nine $P V$ buses and eleven $P Q$ buses. The total system average active and reactive power loads are 1 $383 \mathrm{MW}$ and 453 Mvar, respectively. The peak active and reactive power loads are $2355 \mathrm{MW}$ and $639 \mathrm{Mvar}$, respectively. $3 \times 200 \mathrm{MW}$ units are connected at Bus 2, $4 \times 300 \mathrm{MW}$ units are connected at Bus 3, and a 600-MW unit is connected at Bus 15 . The reliability parameters for generators and transmission lines are shown in Tables A1

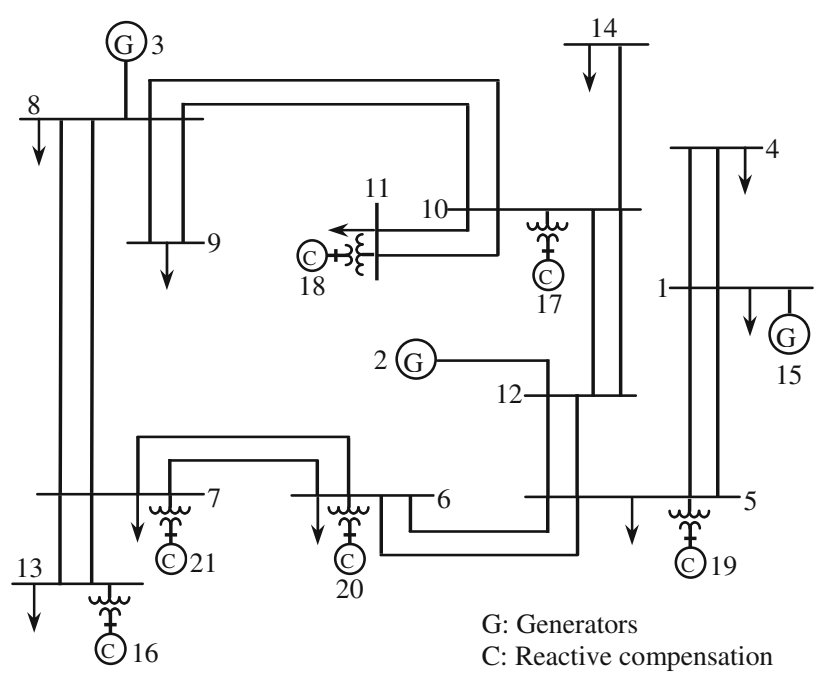

Fig. 4 Simplified 220 kV Taiyuan power system

and A2. The reactive power limits for generators and compensators are shown in Table A1. The states up to the second-order failures have been considered. The voltage set point is assumed to be 0.95 p.u. for the normal state and the first-order failures, and 0.85 p.u. for the second-order failures [25]. The different load-shedding schemes are applied and the corresponding results are compared to illustrate the impact of real and reactive power on system and load point reliability.

\subsection{Real and reactive power load sheddings}

The proposed three-stage load-shedding technique is used to determine comprehensible reliability indices. The real and reactive power loads at each bus are bundled together using the initial power factor in the simulation. The load point, system $E E N S_{P}$, and $E E N S_{Q}$ for the average and peak loads are shown in Table 1.

It can be seen from Table 1 that there are no real and reactive power shortages for average load when considering up to the second-order failures. The $E E N S_{P}$ and $E E N S_{Q}$ at some buses are mainly due to load isolation in transmission line failures. When the peak load is considered, the system will have significant real and reactive power shortages. Bus 1 has the highest $E E N S_{P}$ because the load is $27.6 \%$ of the total system load. Bus 1 also contributes to $85.96 \%$ of the total system $E E N S_{Q}$ because of reactive shortage at this bus.

Table 2 shows the load point, system $E V N S_{P}$ and $E V N S_{Q}$ due to the real power and reactive power shortages. Although the system-expected reactive power curtailment because of the reactive power shortage is smaller than that due to the real power shortage, $E V N S_{Q}$ is much higher than 
Table 1 Load point, system $E E N S_{P}$, and $E E N S_{Q}$ for average and peak load

\begin{tabular}{|c|c|c|c|c|}
\hline \multirow[t]{2}{*}{ Bus } & \multicolumn{2}{|l|}{ Average load } & \multicolumn{2}{|l|}{ Peak load } \\
\hline & $\operatorname{EENS}_{P}\left((\mathrm{MW} \mathrm{h}) \mathrm{a}^{-1}\right)$ & $E E N S_{Q}\left((\mathrm{MW} \mathrm{h}) \mathrm{a}^{-1}\right)$ & $\operatorname{EENS}_{P}\left((\mathrm{MW} \mathrm{h}) \mathrm{a}^{-1}\right)$ & $\operatorname{EENS}_{Q}\left((\mathrm{MW} \mathrm{h}) \mathrm{a}^{-1}\right)$ \\
\hline 1 & 0 & 0 & $151,100.60$ & $223,731.40$ \\
\hline 4 & 0.000444 & 3.388502 & $36,264.16$ & $29,007.67$ \\
\hline 5 & 0 & 0 & $58,348.09$ & $1,218.69$ \\
\hline 6 & 0 & 0 & $20,921.63$ & 436.98 \\
\hline 7 & 0 & 0 & $37,658.93$ & 786.57 \\
\hline 8 & 0 & 0 & $49,979.44$ & $1,043.90$ \\
\hline 9 & 0.000081 & 0 & $20,921.60$ & 436.98 \\
\hline 10 & 0 & 0 & $62,997.35$ & $1,315.80$ \\
\hline 11 & 0.004421 & 0 & $60,440.26$ & $1,266.50$ \\
\hline 13 & 0.000214 & 0 & $26,733.19$ & 558.37 \\
\hline 14 & 6.996100 & 0 & $22,142.35$ & 461.26 \\
\hline System & 7.001300 & 3.388502 & $547,507.60$ & $260,264.10$ \\
\hline
\end{tabular}

Table 2 Load point, system $E V N S_{P}$ and $E V N S_{Q}$ for average and peak loads

\begin{tabular}{|c|c|c|c|c|}
\hline \multirow[t]{2}{*}{ Bus } & \multicolumn{2}{|l|}{ Average load } & \multicolumn{2}{|l|}{ Peak load } \\
\hline & $E V N S_{P}\left((\right.$ Mvar h $\left.) \mathrm{a}^{-1}\right)$ & $E V N S_{Q}\left((\right.$ Mvar h $\left.) \mathrm{a}^{-1}\right)$ & $\operatorname{EVNS}_{P}\left((\mathrm{Mvar} \mathrm{h}) \mathrm{a}^{-1}\right)$ & $E V N S_{Q}\left((\right.$ Mvar h $\left.\left.) \mathrm{a}^{-1}\right)\right)$ \\
\hline 1 & 0 & 0 & $69,738.81$ & $103,260.65$ \\
\hline 4 & 0.000060 & 0.456145 & $4,881.70$ & $3,904.88$ \\
\hline 5 & 0.000016 & 0 & $12,320.52$ & 257.33 \\
\hline 6 & 0 & 0 & $4,649.31$ & 97.11 \\
\hline 7 & 0 & 0 & $6,276.52$ & 131.09 \\
\hline 8 & 0 & 0 & $11,158.20$ & 233.06 \\
\hline 9 & 0 & 0 & $4,184.31$ & 87.40 \\
\hline 10 & 0 & 0 & $11,623.14$ & 242.77 \\
\hline 11 & 0.000982 & 0 & $11,158.21$ & 233.81 \\
\hline 13 & 0.000060 & 0 & $8,136.22$ & 169.94 \\
\hline 14 & 1.499200 & 0 & $4,428.53$ & 92.25 \\
\hline System & 1.500300 & 0.456145 & $148,555.47$ & $108,710.29$ \\
\hline
\end{tabular}

$E V N S_{P}$ at Bus 1. This shows the severe reactive power shortage at Bus 1 .

The results from Tables 1 and 2 show that both real and reactive power shortages are sufficient to supply system average load. If the system operates at its peak level, there is significant shortage of real and reactive power when considering system failures.

\subsection{Real power load shedding}

The total system and load point EENS calculated by the proposed three-stage method (Method 1) are compared to those obtained using conversional proportional real power load shedding (Method 2). The results are shown in Table 3. It can be seen that the load shedding using Method 1 is more reasonable.

\subsection{Reactive power injection}

Reactive power injections at some system locations can solve the voltage problems caused by local reactive power shortage. Because load shedding of real and reactive power usually come together with fixed power factor-based requirements from particular loads, the real power has to be curtailed unnecessarily. Therefore, this article investigates the impact of local reactive power injection to solve the local reactive power shortage problems. The objective of reactive power injection is the same as the load shedding, which is to restore voltage at each bus to its low limit. Table 4 shows load point and system EVarS due to local reactive power shortage or voltage violations for the average and peak load.

Table 4 clearly shows that reactive power shortage at Bus 1 during the peak load is significant even after the reactive 
Table 3 Load point and system EENS for peak load

\begin{tabular}{lrr}
\hline Bus & \multicolumn{2}{c}{ EENS $\left((\mathrm{MW} \mathrm{h}) \mathrm{a}^{-1}\right)$} \\
\cline { 2 - 3 } & \multicolumn{1}{l}{ Method 1 } & \multicolumn{1}{c}{ Method 2 } \\
\hline 1 & $374,832.00$ & $2,533,502.60$ \\
4 & $65,271.83$ & $464,232.40$ \\
5 & $59,566.78$ & $876,604.03$ \\
6 & $21,358.61$ & $278,045.82$ \\
7 & $38,445.50$ & $649,654.85$ \\
8 & $51,023.34$ & $862,196.25$ \\
9 & $21,358.58$ & $195,172.29$ \\
10 & $64,313.15$ & $1,086,768.30$ \\
11 & $61,706.76$ & $572,321.93$ \\
13 & $27,291.56$ & $249,386.81$ \\
14 & $22,603.61$ & $206,073.73$ \\
System & $807,771.70$ & $7,973,959.00$ \\
\hline
\end{tabular}

Table 4 Load point and system EVarS

\begin{tabular}{llc}
\hline Bus & \multicolumn{2}{l}{$E \operatorname{Var} S\left((\right.$ Mvar h $\left.) \mathrm{a}^{-1}\right)$} \\
\cline { 2 - 3 } & Average load & Peak load \\
\hline 1 & 0 & $165,170.68$ \\
4 & 0 & $8,031.40$ \\
5 & 0 & 0 \\
6 & 0 & 0 \\
7 & 0 & 0 \\
8 & 0 & 0 \\
9 & 0 & 0 \\
10 & 0 & 0 \\
11 & 0 & 1.53 \\
13 & 0 & 0 \\
14 & 0 & 0 \\
System & 0 & $173,203.62$ \\
\hline
\end{tabular}

power load is curtailed in the first and second stages. This information is useful for power system planner to select optimal location of future reactive power compensators.

\section{Conclusion}

This article comprehensively investigates reliability issues from both real and reactive power aspects on power system reliability risk evaluation. A three-stage loadshedding process is adopted to evaluate the load point and system reliability of power systems. The reliability indices due to reactive power shortage are compared to those due to real power shortage. The system and local range reactive power shortages are separately solved using different loadshedding methods. Local reactive shortage is also studied using reactive power injection at the nodes with the voltage violation to provide more information for system planning and operation. The equivalent $220 \mathrm{kV}$ Taiyuan system of Shanxi Province in China is analyzed to illustrate the technique. The results show that the reactive power has significant impact on system reliability risk analysis. The load curtailments can be significantly reduced if the detailed reasons of system problems are studied. The reliability indices provide important information for system planners and operators to make their decisions.

Acknowledgments This study was supported by International S\&T Cooperation of China (No. 2010DFB63200) and Shanxi Province Scientific Technological Tackling Key Projects (20130321027-01, 20120321005-02) and Shanxi Province Science and Technology Activities Project for the Selected Scholar Back from Overseas Study.

Open Access This article is distributed under the terms of the Creative Commons Attribution License which permits any use, distribution, and reproduction in any medium, provided the original author(s) and the source are credited.

\section{Appendix}

Table A1 Reliability parameters and reactive power limits

\begin{tabular}{lcclll}
\hline & Bus & $Q_{\min }$ & $Q_{\max }$ & $\lambda$ & $\mu$ \\
\hline Generator & 1 & -20 & 25 & 6 & 194.67 \\
& 2 & -20 & 20 & 4.5 & 219 \\
Compensator & 5 & -20 & 25 & 6 & 194.67 \\
& 8 & -10 & 25 & 6 & 194.67 \\
& 11 & -6 & 20 & 6 & 194.67 \\
& 13 & -6 & 20 & 6 & 194.67 \\
\hline
\end{tabular}

Table A2 Reliability parameters of transmission lines

\begin{tabular}{lccc}
\hline From bus & To bus & $\lambda$ & $\mu$ \\
\hline 1 & 2 & 1 & 876 \\
1 & 3 & 1 & 876 \\
2 & 4 & 1 & 876 \\
3 & 4 & 1 & 876 \\
2 & 5 & 1 & 876 \\
2 & 6 & 1 & 876 \\
4 & 6 & 1 & 876 \\
5 & 7 & 1 & 876 \\
6 & 7 & 1 & 876 \\
6 & 8 & 1 & 876 \\
6 & 9 & 1 & 876 \\
6 & 10 & 1 & 876 \\
9 & 11 & 1 & 876 \\
\hline
\end{tabular}


Table A2 continued

\begin{tabular}{|c|c|c|c|}
\hline From bus & To bus & $\lambda$ & $\mu$ \\
\hline 9 & 10 & 1 & 876 \\
\hline 4 & 12 & 1 & 876 \\
\hline 12 & 13 & 1 & 876 \\
\hline 12 & 14 & 1.5 & 876 \\
\hline 12 & 15 & 1.5 & 876 \\
\hline 12 & 16 & 1.5 & 876 \\
\hline 14 & 15 & 1.5 & 876 \\
\hline 16 & 17 & 1.5 & 876 \\
\hline 15 & 18 & 1.5 & 876 \\
\hline 18 & 19 & 1.5 & 876 \\
\hline 19 & 20 & 1.5 & 876 \\
\hline 10 & 20 & 1.5 & 876 \\
\hline 10 & 17 & 5 & 876 \\
\hline 10 & 21 & 5 & 876 \\
\hline 10 & 22 & 5 & 876 \\
\hline 21 & 22 & 5 & 876 \\
\hline 15 & 23 & 5 & 876 \\
\hline 22 & 24 & 1.5 & 876 \\
\hline 23 & 24 & 1.5 & 876 \\
\hline 24 & 25 & 1.5 & 876 \\
\hline 25 & 26 & 5 & 876 \\
\hline 25 & 27 & 5 & 876 \\
\hline 28 & 27 & 1.5 & 876 \\
\hline 27 & 29 & 5 & 876 \\
\hline 27 & 30 & 5 & 876 \\
\hline 29 & 30 & 5 & 876 \\
\hline 8 & 28 & 1.5 & 876 \\
\hline 6 & 28 & 1 & 876 \\
\hline
\end{tabular}

\section{References}

[1] Taylor CW (1994) Power system voltage stability. McGraw-Hill Inc, New York

[2] Leonardi B, Ajjarapu V (2008) Investigation of various generator reactive power reserve (GRPR) definitions for online voltage stability/security assessment. In: Proceedings of the IEEE Power and Energy Society general meeting: Conversion and delivery of electrical energy in the 21 st century (PES'08), Pittsburgh, PA, USA, 20-24 July 2008

[3] El-Samahy I, Bhattacharya K, Caizares C et al (2008) A procurement market model for reactive power services considering system security. IEEE Trans Power Syst 23(1):137-149

[4] Dong F, Chowdhury BH, Crow ML et al (2005) Improving voltage stability by reactive power reserve management. IEEE Trans Power Syst 20(1):338-345

[5] Hao S, Papalexopoulos A (1997) Reactive power pricing and management. IEEE Trans Power Syst 12(1):95-104

[6] Rajabi A, Monsef H (2007) Valuation of dynamic reactive power based on probability aspects of power system. In: Proceedings of the 42nd international universities power engineering conference (UPEC'07), Brighton, UK, 4-6 September 2007, pp 1169-1174

[7] Tamimi A, Pahwa A, Starrett S (2012) Effective wind farm sizing method for weak power systems using critical modes of voltage instability. IEEE Trans Power Syst 27(3):1610-1617

[8] Yan R, Saha TK (2012) Investigation of voltage stability for residential customers due to high photovoltaic penetrations. IEEE Trans Power Syst 27(2):651-662

[9] Allan RN, Billinton R, Breipohl AM et al (1994) Bibliography on the application of probability methods in power system reliability evaluation: 1987-1991. IEEE Trans Power Syst 9(1):41-49

[10] Allan RN, Billinton R, Breipohl AM et al (1999) Bibliography on the application of probability methods in power system reliability evaluation. IEEE Trans Power Syst 14(1):51-57

[11] Billinton R, Fotuhi-Firuzabad M, Bertling L (2001) Bibliography on the application of probability methods in power system reliability evaluation: 1996-1999. IEEE Trans Power Syst 16(4):595-602

[12] Ding Y, Wang P (2006) Reliability and price risk assessment of a restructured power system with hybrid market structure. IEEE Trans Power Syst 21(1):108-116

[13] Feng Z, Ajjarapu V, Maratukulam DJ (1998) A practical minimum load shedding strategy to mitigate voltage collapse. IEEE Trans Power Syst 13(4):1285-1291

[14] Taylor CW (1992) Concepts of undervoltage load shedding for voltage stability. IEEE Trans Power Deliver 7(2):480-488

[15] Abed AM(1999) WSCC voltage stability criteria, undervoltage load shedding strategy, and reactive power reserve monitoring methodology. In: Proceedings of the IEEE Power Engineering Society summer meeting, vol 2, Edmonton, Canada, 18-22 July 1999, pp 191-197

[16] Zhong J, Nobile E, Bose A et al (2004) Localized reactive power markets using the concept of voltage control areas. IEEE Trans Power Syst 19(3):1555-1561

[17] Moors C, Lefebvre D, Van Cutsem T (2008) Design of load shedding schemes against voltage instability. In: Proceedings of the IEEE Power Engineering Society winter meeting, vol 2, Singapore, 23-27 January 2000, pp 1495-1500

[18] Saadat H (1999) Power system analysis. McGraw-Hill, New York

[19] Qin W, Wang P, Han X et al (2011) Reactive power aspects in reliability assessment of power systems. IEEE Trans Power Syst 26(1):85-92

[20] System Dynamic Performance Subcommittee of the IEEE Power System Engineering Committee (1990) Voltage stability of power systems: Concepts, analytical tools and industry experience. IEEE Publication no 90-THO358-2-PWR

[21] Chen Y (1996) Weak bus-oriented optimal multi-objective var planning. IEEE Trans Power Syst 11(4):1885-1889

[22] Wang P, Billinton R (2000) Optimal load shedding technique to reduce the total customer interruption cost in distribution system. IEE Proc: GenerTransm Distrib 147(1):51-56

[23] Hao S, Papalexopoulos A (1997) Reactive power pricing and management. IEEE Trans Power Syst 12(1):95-104

[24] Rajabi A, Monsef H (2007) Valuation of dynamic reactive power based on probability aspects of power system. In: Proceedings of the 42nd international universities power engineering conference(UPEC'07), Brighton, UK, 4-6 September 2007, pp 1169-1174

[25] Van Cutsem T, Moisse C, Mailhot R (1999) Determination of secure operating limits with respect to voltage collapse. IEEE Trans Power Syst 14(1):327-335 


\section{Author Biographies}

Wenping QIN received her B.Sc. and M.Sc. degrees from Taiyuan University of Technology, China, in 1995 and 2001, respectively. Currently, she is an associate professor in Taiyuan University of Technology. Her interests include power system reliability assessment, stability and security analysis, and power system protection.

Peng WANG (M'00) received his B.Sc. degree from Xi'an Jiaotong University, China, in 1978, the MSc degree from Taiyuan University of Technology, China, in 1987, and the MSc and PhD degrees from the University of Saskatchewan, Canada, in 1995 and 1998 , respectively. Currently, he is an associate professor of Nanyang Technological University, Singapore.
Xiaoqing HAN received her B.Sc., M.Sc., and Ph.D. degrees from Taiyuan University of Technology, China, in 1985, 1990, and 2009, respectively. Currently, she is a professor in Taiyuan University of Technology. Her research interests include power system simulation, power system stability analysis, and grid-connected wind generation analysis. She received Science and Technology Awards of Shanxi province in 2006 and 2009, respectively.

Fanhua MENG received his B.Sc. degree from Tianjin University of Technology and Education, China, in 2010. Currently, he is a postgraduate student in Taiyuan University of Technology. 